\title{
TAGUNG
}

\section{Alternativen zum EU-Beitritt: Die Nachbarschaftspolitik der Europäischen Union am Beispiel von Georgien, Moldova und der Ukraine}

\author{
Ann Gäbler*
}

Die Nachbarschaftspolitik der Europäischen Union war Gegenstand einer deutschsprachigen Auslandstagung des Arbeitskreises für Europäische Integration und des Europäischen Graduiertenkollegs der Deutschen Forschungsgemeinschaft getragen von den Universitäten Heidelberg, Krakau und Mainz an der Jagiellonen-Universität in Krakau. PeterChristian Müller-Graff führte in den Tagungsgegenstand ein. Die Nachbarschaftspolitiken böten osteuropäischen Staaten ohne konkrete Beitrittsperspektive, wie der Ukraine, Moldova und Georgien, Alternativen zur Erweiterungspolitik der Europäischen Union. Sie ermöglichten im Rahmen von bilateralen Partnerschaftsabkommen die Vertiefung der wirtschaftlichen, politischen und kulturellen Zusammenarbeit mit der Europäischen Union in privilegierter Form.

\section{Die EU und ihr Verhältnis zu beitrittswilligen} Nachbarstaaten

Andreas Herdina erläuterte den Rechtsrahmen von Europäischer Gemeinschaft und Europäischer Union für die Europäische Nachbarschaftspolitik. Dazu zeichnete Herdina die bisherige Entwicklung der Europäischen Union nach und zeigte auf, dass die Nachbarschaftspolitik - ein Kind der EU-Erweiterungen in den Jahren 2004 und 2007 - gut, wenn auch mit unterschiedlicher Geschwindigkeit voranschreite. Jedoch betonte Herdina, dass weitere Schritte notwendig seien. Er verwies unter anderem darauf, dass die wirtschaftliche Integration der Nachbarn verstärkt werden

\section{Die Nachbarschaftspolitik der Europäischen Union gegenüber beitrittswilligen osteuropäischen Demokratien}

Tagung des Arbeitskreises Europäische Integration und des Europäischen Graduiertenkollegs der Universitäten Heidelberg, Krakau und Mainz

Krakau, 15.-16. Juni 2007

\section{Wissenschaftliche Leitung}

Prof. Dr. Dr. h.c. Kazimierz LANKOSZ, Universität Krakau

Prof. Dr. Dr. h.c. Peter-Christian MÜLLERGRAFF, Universität Heidelberg

Prof. Dr. Udo FINK, Universität Mainz

Einführung

Prof. Dr. Dr. h.c. Peter-Christian MÜLLERGRAFF, Universität Heidelberg

Die Europäische Nachbarschaftspolitik im Rechtsrahmen von EG/EU - Instrument gegenüber Nachbarn in Europa

Andreas HERDINA, Europäische Kommission, Brüssel

Prof. Dr. Tadeusz WŁUDYKA, Universität Krakau (Statement)

Die Partnerschaftsabkommen mit osteuropäischen Nachbarstaaten

Prof. Dr. Dr. h.c. Kazimierz LANKOSZ, Universität Krakau

Neuschaffung von Privatrechtsordnungen in Georgien, Moldova und der Ukraine

Professor Dr. Dr. h.c. Rolf KNIEPER, Universität Bremen

Prof. Dr. Fryderyk ZOLL, Universität Krakau (Statement)

* Ann Gäbler, Wissenschaftliche Mitarbeiterin, Institut für deutsches und europäisches Gesellschafts- und Wirtschaftsrecht, Universität Heidelberg. 
müsse, langfristig sei mittels vertiefter Freihandelsabkommen eine Nachbarschafts-Wirtschaftsgemeinschaft anzustreben. Auch sollten die bilateralen Beziehungen $\mathrm{zu}$ den östlichen Nachbarn durch multilaterale Verhandlungen ergänzt werden, um sektorübergreifende Themen verstärkt angehen $\mathrm{zu}$ können. Weiterhin betonte Herdina die Bedeutung der Schwarzmeersynergie-Initiative als Mittel zur Intensivierung des Dialoges der Europäischen Union und den Ländern der Region. Zudem müssten die schwierigen Fragen des Visa-Rechts gelöst und der zivilgesellschaftliche Austausch, etwa in Form von gemeinsamen Bildungsmaßnahmen und Austauschprogrammen intensiviert werden. Sodann wandte sich Herdina der Frage der Rechtsnatur der Nachbarschaftspolitik zu. Diese sei Außenpolitik und dennoch Politik sui generis, rechtliches Fundament bildeten die bestehenden Abkommen. Zuletzt beleuchtete Herdina, in welchem Maße die Nachbarstaaten ihren Rechtsbestand an den der Europäischen Union anzugleichen haben. Zwar sei $\mathrm{zu}$ beobachten, dass eine Rechtsübernahme stattfinde, allerdings werde anders als im Rahmen der Beitrittspolitik keine pauschale Rechtsangleichung verlangt, dies sei in gewissen Bereichen wie dem des Umweltschutzes auch unrealistisch.

Tadeusz Włudyka wies darauf hin, dass Polen als Mitgliedstaat an der Grenze der Europäischen Union unmittelbar von der Nachbarschaftspolitik betroffen sei. Er betonte die Anziehungskraft der Europäischen Union, bereits die potenzielle Möglichkeit eines Beitritts habe etwa in der Ukraine und in Georgien die Reformwilligkeit stark gesteigert, weshalb ein Beitritt nicht grundsätzlich ausgeschlossen werden sollte. Aus wirtschaftlicher Sicht müsse die Regionalentwicklung der Grenzbereiche unterstützt werden, um ein Auseinanderklaffen der Lebensbedingungen zu vermeiden. Als konkrete Maßnahmen schlug Włudyka unter anderem die Erleichterung des Grenzübertritts für Bewohner von Grenzbereichen und Investitionen in die Infrastruktur vor. Zusammenfassend plädierte
Rechtsstaatliche Rahmenbedingungen für eine Justiz im Lichte der Systemtransformation in Georgien

Prof. Dr. Lado CHANTURIA, Direktor des Projekts Zivil- und Wirtschaftsrecht im Kaukasus und in Zentralasien, Universität Bremen

Recht und Praxis der Wirtschaftsordnung: Marktwirtschaft und Wettbewerbsfähigkeit in der Ukraine

Prof. Dr. Wilfried TRILLENBERG, Wirtschaftsuniversität Ternopil/Ukraine

Angleichung an das Recht der EG in Osteuropa im Wettbewerb mit amerikanischen Modellen Prof. Dr. Helmuth SCHRÖTER, Universität des Saarlandes, Europäische Kommission Brüssel

Prof. Dr. Jerzy PISULIŃSKI, Universität Krakau (Statement)

Institutionalisierte Zusammenarbeit zwischen Grenzregionen der Ukraine und EU-Mitgliedstaaten

Prof. Dr. Irena LIPOWICZ, Universität Warschau

Dr. Ulrich ERNST, Universität Krakau (Statement)

Visa- und Migrationsrecht im Verhältnis zwischen EU und osteuropäischen Nachbarn

Christian KOVÁCS, Andrássy-Universität Budapest

Dr. Marek PORZYCKI, Universität Krakau (Statement)

Rechtliche Bindungen im Rahmen alternativer Integrationskonzepte: GUS, GUAM und Einheitlicher Wirtschaftsraum

Łukasz ADAMSKI, Polnisches Institut für internationale Angelegenheiten (PISM), Warschau

Włudyka für eine spezifische Nachbarschaftspolitik zur Entwicklung der Grenzregionen im Osten, welche keinesfalls versäumt werden dürfe.

Kazimierz Lankosz betonte die Verantwortung der Europäischen Union: Der hohe Lebensstandard und das friedliche Miteinander dürften nicht an den Grenzen der Europäischen Union enden. Lankosz qualifizierte die Nachbarschaftspolitik als Grundlage für die 
Schaffung besonderer Beziehungen zu einem Drittstaat und als Mittel zur Verhinderung weiterer Grenzziehung und der Ermöglichung von Wohlstand und Sicherheit innerhalb Europas. Da wirtschaftliche Entwicklung letztlich größere Stabilität in Europa bewirke, sei Nachbarschaftshilfe Unterstützung für die Nachbarstaaten, aber auch vorteilhaft für die Europäische Union selbst. Sie müsse daher attraktive Alternativen schaffen, um reformwilligen Nachbarländern zu helfen. Dazu sollten die vorhandenen bilateralen Instrumente ausgebaut werden, Schlüsselelemente bildeten insoweit die bilateralen Aktionspläne als Träger der politischen und wirtschaftlichen Agenda. Andere gemeinsame Interessen, wie das öffentliche Gesundheitswesen, der Grenzschutz, Energie- und Migrationsfragen, müssten hingegen zum Gegenstand multilateraler Abkommen gemacht werden.

Die Rechtsentwicklung in Georgien, Moldova und der Ukraine seit der politischen Wende

Rolf Knieper skizzierte den intrinsischen Prozess der Reformen der Privatrechtsordnungen dieser Länder. Nach der Auflösung der Sowjetunion seien von den Nachfolgestaaten zunächst Verfahren zur Gestaltung von Modellgesetzen initiiert worden, die auch die Neugestaltung eines - von einer russischen Zivilrechtsdoktrin stark beeinflussten - Zivilgesetzbuches (ZGB) unternahmen. Inhaltlich kritisiert worden sei dieses Modellgesetz etwa wegen der scharfen Haftungsregelungen zum Nachteil von gewerblichen Unternehmen und der Beibehaltung von Vertragstypen, die den marktwirtschaftlichen Gegebenheiten widersprächen. Bemängelt worden seien zudem die Gesetzgebungsmethode, die allgemeine $\mathrm{Ge}$ setzessystematik und die lehrbuchartigen $\mathrm{Er}$ läuterungen. Georgien, Moldova und die Ukraine hätten daher, wenn auch in unterschiedlichem Maße, abweichende Zivilgesetzbücher geschaffen. Knieper erläuterte sodann einige Details der Entstehungsgeschichte, Methodik und Technik der materiell-rechtlichen Regelungen. Problematisch sei bereits die Frage gewesen, welche Regelungs- materien im jeweiligen ZGB Platz finden sollten. Sehr ausführlich und kritisch widmete sich Knieper der Darstellung des ukrainischen Wirtschaftskodex. Weitere Problempunkte seien die Möglichkeit eines Vorrangs des ZGB und die Kodifikationstechnik im Allgemeinen, letztere führte in der Ukraine und Moldova sehr oft zu Doppelungen und Widersprüchlichkeiten. Abschließend zeigte Knieper detailliert die Gemeinsamkeiten und Unterschiede der Zivilgesetzbücher aller drei Länder auf. Zusammenfassend kam er zu dem Ergebnis, dass die betroffenen Privatrechtsordnungen trotz ihrer Entstehung im Rahmen einer Übergangsphase auf gesetzliche Stabilität und Rechtssicherheit gerichtet seien.

Fryderyk Zoll beleuchtete die polnische Perspektive zu Privatrechtsreformen. Polen habe, nicht zuletzt wegen seiner geografischen Lage in der Mitte zwischen Osten und Westen, mit ähnlichen Problemen zu ringen gehabt. Diese Prozesse seien für den Aufbau einer Demokratie unvermeidbar. Das Privatrecht habe neu an Bedeutung gewonnen. Nach Zolls Einschätzung sei Polen auf einem guten Weg, aufgrund der gefestigten Rechtstradition seien die Ergebnisse der juristischen Forschung und die Grundstrukturen des Privatrechts erhalten geblieben. Das russische Privatrecht bezeichnete Zoll als „Oase des Kontaktes mit der römischen Rechtstradition“. So habe das Privatrecht vor der politischen Wende eine wesentliche Rolle als Traditionshalter für eine normale Welt gedient. Abschließend warf Zoll verhalten die Frage nach der Kodifikation eines europäischen Privatrechts auf.

Lado Chanturia widmete sich den rechtsstaatlichen Rahmenbedingungen für eine Transformation der Justiz in Georgien. Eine Stärkung der Rechtsstaatlichkeit sei nur durch eine Reform des öffentlichen Dienstes und des Justizwesens sowie die Verbesserung der demokratischen Kontrollmöglichkeiten zu erreichen. Als wesentlich stellte Chanturia zudem die nationale Strategie zur Korruptionsbekämpfung heraus. Im Einzelnen ging Chanturia auf die Notwendigkeit von unab- 
hängigen und unparteiischen Richtern, die verfassungsrechtlichen Grundlagen der rechtsprechenden Gewalt und die Bedrohungen ein, die sich für die Unabhängigkeit der Justiz ergäben. Letztere könnten sich sowohl aufgrund äußerer Einflüsse (politischem Druck, Manipulation der öffentlichen Meinung, Korruption) als auch interner Gründe (Druck durch obere Gerichtsinstanzen oder den Gerichtspräsidenten) ergeben. Chanturia kam zu dem Ergebnis, dass eine aufgeklärte Gesellschaft und hervorragend ausgebildete Juristen unabdingbare Voraussetzungen für die Verwirklichung von Rechtsstaatlichkeit in Form unabhängiger Gerichtsbarkeit seien.

Wilfried Trillenberg äußerte sich zur Marktwirtschaft und Wettbewerbsfähigkeit in der Ukraine aus ökonomischer Sicht und stellte dazu drei Thesen auf. Erstens zeige die Ukraine im Rahmen der Nachbarschaftspolitik der Europäischen Union ihren Beitrittswillen. Dieser Prozess müsse zunehmend in gemeinsamem Handeln Gestalt annehmen. Zweitens forderte Trillenberg eine Prozessdiskussion über die Inhalte der auf beiden Seiten für einen EU-Beitritt notwendigen Handlungen. Drittens betonte er die Notwendigkeit der Schaffung rechtlicher Regelungen zur Verstärkung des marktwirtschaftlichen Unternehmertums und der Wettbewerbsfähigkeit ukrainischer Unternehmen. Zur Verdeutlichung der aktuellen Situation in der Ukraine stellte Trillenberg in Form von Schlagworten die in der Literatur und im Internet geäußerten Ansichten zur Ukraine in den Bereichen der sozioökonomischen Entwicklung, der Markt- und Wettbewerbsordnung, der Investitionsrisiken, der Investitionsempfehlungen und der Lohnkosten dar, um diese mit eigenen Erfahrungen zu vergleichen. Abschließend sprach Trillenberg sich für eine Verbindung von Russland und der Europäischen Union im Bereich des Energiesektors unter Einbeziehung der Ukraine aus.

Helmuth Schröter befasste sich mit der Angleichung des Rechts osteuropäischer Länder an das Recht der Europäischen Gemeinschaft.
Er unterschied insoweit zunächst zwischen der Rechtsangleichung innerhalb der Gemeinschaft, im Bereich des Europäischen Wirtschaftsraums und der Europa-Abkommen einerseits und der Rechtsangleichung im Rahmen von Partnerschaftsabkommen andererseits. Partnerschaftsabkommen seien den Europa-Abkommen zwar in Struktur und Inhalt ähnlich, zielten anders als diese indes nicht auf eine Integration der osteuropäischen Staaten in die Europäische Gemeinschaft. Sie bezweckten vielmehr die Unterstuitzung von Transformationsprozessen; eine stärkere Bindung der betreffenden Länder an die Europäische Gemeinschaft sei aber gewünscht. In diesem Zusammenhang ging Schröter ebenfalls kurz auf die Aktionspläne der Europäischen Nachbarschaftspolitik ein. Abschließend betonte er, dass Moldova, die Ukraine und Weißrussland sich bei ihrer Gesetzgebung sehr stark an den gemeinschaftsrechtlichen Vorbildern orientierten. Eine Beeinträchtigung der Hinwendung der osteuropäischen Staaten zum Rechts- und Wirtschaftssystem der Europäischen Union durch die Konkurrenz amerikanischer Modelle sei daher - trotz der starken Präsenz der USA - nicht zu befürchten.

Mit seinem Statement zu den polnischen Erfahrungen bei der Transformation des polnischen Rechts zur Rechtsangleichung schloss Jerzy Pisuliński den ersten Konferenztag. Der Schwerpunkt der Gesetzgebung habe nach dem politischen Umbruch auf der Schaffung einer neuen privatwirtschaftlich orientierten Rechtsordnung gelegen. In der Folge sei es 1994 zum Erlass des neuen polnischen Zivilgesetzbuches gekommen, welches allerdings auch viele Institute des ZGB von 1964 übernehmen konnte. Weitere Kodifikationen, wie das Gesetz über wirtschaftliche Tätigkeit, das Devisengesetz, das Gesetz über die Privatisierung staatlicher Unternehmen und das Gesetz über den unlauteren Wettbewerb, hätten den neuen Rahmen für privatwirtschaftliche Tätigkeit gebildet. Aufgrund der Beitrittsbestrebungen Polens sei die Gesetzgebung in der Folge sehr stark vom Ziel der Rechtsanglei- 
chung an das EG-Recht geprägt, der Einfluss des amerikanischen Rechts deshalb gering gewesen.

\section{Einzelaspekte der Nachbarschaftspolitik der Europäischen Union}

Irena Lipowicz wies auf die Situation der Regionen in Polen hin. Sie machte deutlich, dass vor der politischen Wende selbst der Begriff der kommunalen Selbstverwaltung verboten war, diese sich jedoch seit 1990 in Form der Gemeinden und seit 1998 in Form der Kreise und Wojewodschaften sehr gut wieder entwickelt habe und auch im Bereich der grenzüberschreitenden Zusammenarbeit ein hohes Potenzial aufweise. Die Zusammenarbeit entwickle sich auch im polnisch-ukrainischen Bereich, Schwierigkeiten bereite jedoch die Konstruktion und Praxis der Euroregionen an den Grenzen zur Ukraine. Lipowicz führte dies unter anderem auf das Trauma der Teilung und den Bürgerkrieg zurück. Die Barrieren zeigten sich etwa darin, dass es nur sieben Grenzübergänge gibt. Die Region Podkarpacki habe auf polnischer Seite keine alte Regionalmetropole, Rzeszow habe erst begonnen, diese Rolle auszufüllen. Auf ukrainischer Seite komme hinzu, dass der Freiheitsund Regionalgedanke in den Köpfen der Menschen erst sehr viel später auch Realität wurde, weshalb die Zusammenarbeit in der Euroregion Podkarpacki erschwert sei. Sowohl auf polnischer als auch auf ukrainischer Seite seien die Grenzregionen schwach entwickelt. Es gäbe jedoch auch Chancen: Das große Potenzial der Grenzregionen sei die Intelligenz, Individualität und Kreativität der Menschen, die in den polnisch-ukrainischen Grenzregionen leben. Notwendig sei jedoch gegenseitiger Respekt und Zusammenarbeit auf gleicher Augenhöhe sowie die Beseitigung der vorhandenen Vorurteile. Besonders gut funktionierten daher Projekte, die Bildung und Regionalentwicklung fördern. Die grenzüberschreitende Zusammenarbeit dürfe allerdings nicht nur auf die regionalen Kräfte bauen. Helfen könne insoweit die trilaterale Zusammenarbeit mit der Slowakei unter Ein- beziehung Deutschlands. Für die Zukunft wünschte sich Lipowicz die Dezentralisierung der vorhandenen großen Regionen.

Ulrich Ernst schilderte den schwelenden Transnistrienkonflikt. Nach einem kurzen historischen Rückblick und der Darstellung der derzeitigen Konfliktlage, zeigte Ernst denkbare Möglichkeiten der Auflösung des Konfliktes unter Ausklammerung geopolitischer Hintergründe auf. Denkbar sei zunächst die Vereinigung Moldovas ohne Transnistrien mit Rumänien und die Einbindung Transnistriens in die Ukraine. Dagegen sprächen jedoch die Frage der slawischen Minderheit in „Restmoldova“ und das Schicksal der Gagausen sowie der hohe völkerrechtliche Aufwand. Nicht auszuschließen sei daher die Beibehaltung der Republik Moldova als Einheitsstaat mit europäischer Perspektive, entweder unter Gewährleistung der transnistrischen Autonomie oder mittels der Gestaltung der Republik Moldova mit den Bundesstaaten „Transnistrien“ und „Restmoldova“. Aus Sicht der Europäischen Union sei es notwendig, die EU-Außengrenze in Rumänien zu sichern. Ernst zog daher den Aufbau einer Grenze zu Transnistrien und die Einrichtung einer EU-Grenzmission in Betracht.

Dem Visa- und Migrationsrecht im Verhältnis zwischen der Europäischen Union und ihren osteuropäischen Nachbarn widmete sich Christian Kovács am Beispiel der Ukraine. Nach einem Rückblick auf die deutsche Visaaffäre skizzierte Kovács die normativen Grundlagen des Visa- und Migrationsrechts im EG-Vertrag und im Sekundärrecht, um diese anschließend im Hinblick auf Osteuropa zu analysieren. Das europäische Migrationsrecht sei vor allem restriktiv ausgestaltet, enthalte es doch überwiegend Regelungen zur Verhinderung von legaler und Bekämpfung illegaler Einwanderung. Negative Auswirkungen habe dies für die östlichen Nachbarstaaten der Europäischen Union. Anschaulich zeige sich dies am Beispiel des Verhältnisses zwischen Polen und der Ukraine. Visaerleichterungen für die Ukraine seien bislang im 
Rahmen von Rücknahmeabkommen vorgesehen, eine Befreiung von der Visapflicht sei jedoch nicht abzusehen. Kovács warb abschlieBend für eine Liberalisierung der Regelungen zur Wirtschaftsmigration.

Marek Porzycki schilderte die Situation an der polnisch-ukrainischen Grenze, wie sie sich für einen EU-Bürger darstelle. Er qualifizierte die Visapflicht als schädlich für die menschlichen Kontakte und die Zusammenarbeit zwischen Polen und der Ukraine. Aufgrund der schwierigen gemeinsamen Geschichte bestehe zwischen den Ländern ein großer Versöhnungsbedarf, die Aussöhnung werde jedoch durch die Reiseschwierigkeiten erschwert. Auch wirtschaftlich belaste die Visapflicht die betroffenen Ukrainer, welche Visagebühren und Zeit für die Reise zum Konsulat aufbringen müssten. Dies habe mittelbar auch Auswirkungen auf die Europäische Union, da der ukrainische Mittelstand diese wegen der Visapflicht meide. Porzycki sprach sich daher für eine Abschaffung der Visapflicht im Rahmen einer langfristigen Nachbarschaftspolitik aus. Gerade ihre flexible bilaterale Natur ermögliche es, für jeden Staat geeignete individuelle Visaerleichterungen zu finden. Letztlich erfordere dies allerdings den Abbau negativer Assoziationen bezüglich der östlichen Nachbarn.
Abschließend widmete sich Łukasz Adamski den rechtlichen Bindungen der Ukraine im Rahmen alternativer Integrationskonzepte und den damit verbundenen rechtlichen und politischen Auswirkungen auf einen möglichen Beitritt zur Europäischen Union. Trotz ihrer Unabhängigkeitsbestrebungen habe die Ukraine sich aufgrund emotionaler, menschlicher und wirtschaftlicher Verflechtungen mit den anderen postsowjetischen Staaten an verschiedenen Integrationskonzepten beteiligt, etwa im Rahmen der Gemeinschaft Unabhängiger Staaten (GUS), der GUAM ${ }^{1}$ und dem Europäischen Wirtschaftsraum (EWR), ohne jedoch dabei die Möglichkeit eines EU-Beitritts aus den Augen zu verlieren. Einen solchen hielt Adamski unter gleichzeitiger Beteiligung an der GUS grundsätzlich für möglich, notwendig sei indes die Kündigung diverser Vereinbarungen, etwa der zur Koordinierung der Sicherheits- und Außenpolitik. Die Teilnahme der Ukraine am EWR sah Adamski im Hinblick auf einen EU-Beitritt kritisch, handele es sich dabei doch um eine der Europäischen Gemeinschaft im Grunde nachgebildete Struktur minderer Dichte. Dagegen schaffe die Beteiligung der Ukraine an der GUAM keine mit einem EU- und NATO-Beitritt unvereinbaren Verpflichtungen, sie könne die Integration sogar vorantreiben.

1 Sicherheitsallianz der vier GUS-Staaten Georgien, Ukraine, Aserbaidschan und der Republik Moldova. 